\title{
The vignette for V13N5 Issue
}

\author{
Ginsenoside Rb1 inhibits substance $\mathbf{P}$ enhancement \\ of allergen-induced RBL cells
}

Ginseng has been used extensively in traditional herb medicine; however, its mode of action remains elusive. Ginsenoside Rh2 and the metabolite of Rb1 have been shown to have anti-inflammatory activities on LPS-induced macrophages $[1,2]$. In this issue, Liao et al. [3] reported that Rb1 dose-dependently inhibited Substance P- enhanced allergeninduced mediator release. In addition, the authors also presented evidence suggesting that its mechanism is through the inhibition of the ERK pathway. These findings provide additional insight into the mechanism of ginseng as an anti-inflammatory substance.

\section{Direct interaction of $\mathrm{C} / \mathrm{EBP} \delta$ and Sp1 at the $\mathrm{GC}$-enriched promoter region synergizes the IL-10 gene transcription in mouse macrophage}

CCAAT/enhancer-binding protein (C/EBP) family members are basic-leucine-zipper transcription factors which recognize specific DNA sequences as either homodimers or heterodimers [4]. C/EBP factors functionally interact with $\mathrm{Sp} 1$ and participate in the tissue-specific gene expression $[5,6]$. A previous study has shown that LPS activates the transcription of the IL-10 gene through the Sp1 and $\mathrm{C} / \mathrm{EBP}-$ binding sites and indicated that $\mathrm{Sp} 1, \mathrm{C} / \mathrm{EBP} \beta$ and $\mathrm{C} / \mathrm{EBP} \delta$ can coactivate the IL-10 gene expression in mouse macrophage cells [7]. Chiang BT et al [8] showed that $\mathrm{C} / \mathrm{EBP} \delta$ directly interacts with $\mathrm{Sp} 1$ via its basic region leucine zipper domain. This observation provides a mechanistic insight into cooperation of transcriptional factors in regulation of tissue-specific gene expression.

\section{Mapping the antigenic determinants and reducing the immunogenicity of trichosanthin by site-directed mutagenesis}

Trichosanthin (TCS) is a natural protein from root tuber that has been reported to inhibit the Integr- ase enzyme of HIV-1 [9]. TCS inhibits HIV-1 replication in vitro [10] and showed promising results in clinical trials [11]. However, strong immunogenicity of TCS hindered its further development into an anti-HIV drug. Based on the available X-ray structure of the protein, Anetal [12] located the immunogenic domains by modeling, and subsequently mutated key residues. Some mutants were indeed less immunogenic in mice. The mutants remain biologically active (DNase activity, ribosome inactivation), indicating that they fold similarly to the wild-type protein. These modified TCS proteins may be developed further as antiviral.

\section{Mutations in the $\alpha$-helix directly C-terminal to the major homology region of human immunodeficiency virus type 1 capsid protein disrupt Gag multimerization and markedly impair virus particle production}

Retroviral particles are made of multimers of the structural Gag protein, involving Gag-Gag interactions. Chin-Tien Wang previously mapped the Gag domain that is responsible for incorporation of the Gag-Pol fusion protein in HIV-1 particles [13]. They now mapped a small $\alpha$-helical region of the capsid (CA) domain that is important for Gag multimerization and virus particle production [14]. Furthermore, some of the mutations also affect the interaction of Gag with membranes, the location where virion particles are assembled.

\section{Potent Inhibition of Human Telomerase by $\mathrm{U}-\mathbf{7 3 1 2 2}$}

Telomerase is an enzyme critical for maintaining the terminal structure of DNA molecules of nearly all eukaryotic chromosomes [15]. Interestingly, it is inactive in most adult tissues but active in many caner cells and cultured cells. Consequently, telomerase has been an intended target of many anti-cancer drugs [16]. Search for inhibitors of telomerase is thus important to further our under- 
standing of telomerase as well as improving cancer intervention. In this study, the authors have shown the effectiveness of an amphiphilic alkylating agent U-73122 in telomerase inhibition both in cultured cells and cell-free systems [17]. The compound is commonly used as an inhibitor for phospholipase $\mathrm{C}$, in contrast to those predicted to work on telomerase. Its inhibitory mechanism may offer new possibility for clinical applications.

\section{Impairment of transneuronal traffic in Streptozotocin-induced diabetes: a wheat germ agglutinin-horseradish peroxidase neurochemical study in the rat}

Autonomic neuropathy is a fairly common diabetic complication that may result in gastric dysfunction [18, 19]. Pathological changes of the vagus nerve are thought to be associated with such autonomic disorders [20, 21]. Streptozotocininduced diabetes in rats has been shown to alter the neurotrophin content in the cervical vagus nerve [22]. In this issue, based on a decrease in the number of retrogradely labeled neurons within the dorsal motor nucleus of the vagus nerve in Streptozotocin-induced diabetic rats as compared with control euglycaemic rats, it was suggested that the retrograde transport process along the axons of the affected neurons was impaired during chronic diabetes [23]. Although impaired neuronal transport is implicated in the findings of Odekunle [23], further investigation is needed to elucidate whether endogenous neurotrophin is involved in the process.

\section{Redistribution of GFAP and $\alpha \beta$ crystalline after thermal stress in $\mathrm{C} 6$ glioma cell line}

Intermediate filament (IF) proteins expressed in the development of glia include vimentin, nestin and glial fibrillary acidic protein (GFAP). [24] Enhanced green fluorescent protein (EGFP)GFAP transiently expressed in C6 cells was first found in the cytoplasm and then gradually formed a filamentous structure of EGFP-GFAP. The protein level of nestin in C6- EGFP-GFAP stable clone was similar to that in non-transfected C6 cells while the level of vimentin was reduced. Interestingly, the expression level of $\alpha \beta$ crystallin, a small heat shock protein, was up-regulated by heat shock treatment; GFAP formed IF bundles in C6EGFP-GFAP cells, and $\alpha \beta$ crystallin was colocalized with the IF bundles of GFAP. It suggests that $\alpha \beta$ crystallin may regulate the cytoarchitecture of GFAP [25].

\section{Co-administration of Dextromethorphan with methamphetamine attenuates methamphetamine-induced rewarding and behavioral sensitization}

Abuse of methamphetamine (MA) worldwide. increased considerably during the past two decades. MA is a well-known central nervous system stimulant, which produces strong rewarding and behavioral sensitization via activation of the mesolimbic dopaminergic system after repeated administration [26]. Dextromethorphan (DM), as a non-competitive antagonist of the NMDA receptor, effectively attenuates the morphine-induced tolerance and withdrawal syndrome in animal models [27]. Yang et al. reported in this study that co-administration of DM with MA significantly abolished the MA-induced rewarding/ drug seeking behavior and increases in locomotor and stereotypic activities. They further demonstrated that suppression of the MA-induced increase in dopamine turnover rate in the mesolimbic dopaminergic system by DM underlies the inhibitory effect of DM on MA-induced rewarding and behavioral sensitization.

\section{Overdrive and SA node discharge}

Overdrive of cardiac pacemakers is followed by a temporary suppression of discharge which is much shorter in the sino-atrial node (SAN) [28] than in Purkinje fibers [29]. The reason for this difference has not been determined. It might be related to the fact that morphologically and functionally the SAN is not a homogeneous structure, since slow responses are recorded in dominant pacemaker cells and fast responses in subsidiary pacemaker cells [30]. The hypothesis that the pause that follows overdrive of SAN might be the net result of overdrive excitation and overdrive suppression was tested by studying the rate and force patterns induced by overdrive in isolated guinea pig SAN 
superfused in vitro. It is concluded that in SAN the prevalence of $\mathrm{Ca} 2+$ load leads to overdrive excitation whereas the prevalence of $\mathrm{Na}+$ load leads to overdrive suppression. In Tyrode solution, the pause after drive appears to be the net result of these two different mechanisms [31].

\section{Carbon monoxide mediates HO-1-induced protection of intimal hyperplasia}

Heme oxygenase-1 (HO-1) is a stress-inducible enzyme catalyzing the degradation of heme to liberate free ion, carbon monoxide (CO) and biliverdin [32]. The first line of evidence supporting the anti-thrombotic effect of HO-1 in vivo is the observation that $\mathrm{CO}$ suppressed vascular thrombosis occurring during the cardiac graft rejection likely through inhibiting platelet aggregation [33]. Chen et al., [34] in the present study applied an injury model by performing angioplasty in the carotid arteries of the hypercholesterolemic animals, apoE-deficient mice, to assess the effect of adenovirus-mediated HO-1 transduction on the thrombotic response of injured vessels, and evaluated the potential role of $\mathrm{CO}$ in this process. The thrombolytic effect was observed by exposing animals with existing arterial thrombosis to $\mathrm{CO}$. The results support a role of early thrombolysis induced by $\mathrm{CO}$ in $\mathrm{HO}-1$-mediated protection against intimal hyperplasia after vascular injury.

\section{Tetramethylpyrazine induces heme oxygenase-1 expression and attenuates myocardial ischemia/reperfusion injury in rats}

Free radicals formed during ischemia/reperfusion are believed to play a major role in the pathology of cardiac injury [35, 36]. In this issue, Chen et al., reported that the cardioprotective activity of tetramethylpyrazine (TMP), an active ingredient of Chinese medicinal herb Ligusticum wallichii Franchat, may be associated with its antioxidant activity via induction of heme oxygenase-1 and with its capacity for neutrophil inhibition [37]. These findings are significant and may pave the way for further development of therapeutic agents for ischemia/reperfusionrelated cardiac injury.

\section{References}

1. Park E.K., Choo M.K., Kim E.J., Han M.J. and Kim D.H., Antiallergic activity of ginsenoside Rh2. Biol. Pharm. Bull. 26: 1581-1584, 2003.

2. Park E.K., Shin Y.W., Lee H.U., Kim S.S., Lee Y.C., Lee B.Y. and Kim D.H., Inhibitory effect of ginsenoside Rb1 and compound $\mathrm{K}$ on $\mathrm{NO}$ and prostaglandin E2 biosyntheses of RAW264.7 cells induced by lipopolysaccharide. Biol. Pharm. Bull. 28: 652-656, 2005.

3. Liao B.C., Hou R.C.W., Wang J.S. and Jeng K.C.G., Enhancement of the release of inflammatory mediators by substance $\mathrm{P}$ in rat basophilic leukemia RBL-2H3 cells. J. Biomd. Sci. 2006 (this issue doi 10.1007/s11373-006-9099-1).

4. McKnight S. (1992) Transcriptional Regulation, pp. 771-795, Cold Spring Harbor Laboratory Press, Cold Spring Harbor, NY 2, Lo'pez-Rodrı'guez C., Botella L., and Angel L. Corb1 AL, J. Biol. Chem. 272:29120-29126, 1997.

5. Lopez-Rodriguez C., Botella L. and Corbi A.L., CCAATenhancer-binding proteins $(\mathrm{C} / \mathrm{EBP})$ regulate the tissue specific activity of the CD11c integrin gene promoter through functional interactions with Sp1 proteins. J. Biol. Chem. 272: 29120-29126, 1997.

6. Tzeng S.J., Chang W.C. and Huang J.D., Transcriptional regulation of the rat Mrp3 gene promoter by the specificity protein $(\mathrm{Sp})$ family members and CCAAT/enhancer binding proteins. J. Biomed. Sci. 12: 741-761, 2005.

7. Liu Y.W., Tseng H.P., Chen L.C., Chen B.K. and Chang W.C., Functional cooperation of simian virus 40 promoter factor 1 and CCAAT/enhancer-binding protein beta and delta in lipopolysaccharide-induced gene activation of IL-10 in mouse macrophages. J. Immunol. 171: 821-828, 2003.

8. Chiang B.T., Liu Y.W., Chen B.K., Wang J.M. and Chang W.C., Direct interaction of $\mathrm{C} / \mathrm{EBP} \delta$ and $\mathrm{Spl}$ at the GC-enriched promoter region synergizes the IL-10 gene transcription in mouse macrophage. J. Biomed. Sci. (this issue doi 10.1007/s11373-006-9101-y).

9. Au et al., The plant Ribosome inactivating proteins luffin and saporin are potent inhibitors of HIV-1 integrase. FEBS Lett. 471, 169-172, 2000.

10. McGrath M.S., Hwang K.M., Caldwell S.E., Gaston I., Luk K.C., Wu P., Ng V.L., Crowe S., Daniels J. and Marsh J., GLQ223: an inhibitor of human immunodeficiency virus replication in acutely and chronically infected cells of lymphocyte and mononuclear phagocyte lineage. Proc. Natl. Acad. Sci. USA. 86: 2844-2848, 1989.

11. Kahn J.O., Gorelick K.J., Gatti G., Arri C.J., Lifson J.D., Gambertoglio J.G., Bostrom A. and Williams R., Safity, activity, and pharmacokinetics of GLQ223 in patients with AIDS and AIDS-related complex. Antimicrob. Agents Chemother. 38: 260-267, 1994.

12. An Q., Wei S., Mu S., Zhang X., Lei Y., Zhang W., Jia N., Cheng X., Fan A., Li Z. and Xu Z., Mapping the antigenic determinants and reducing the immunogenicity of trichosanthin by site-directed mutagenesis. J. Biomed. Sci. (this issue doi 10.1007/s11373-006-9095-5).

13. Chiu H.C., Liao W.H., Chen S.W. and Wang C.T., The human immunodeficiency virus type 1 carboxyl-terminal third of capsid sequence in Gag-Pol is essential but not sufficient for efficient incorporation of Pr160gag-pol into virus particles. J. Biomed. Sci. 11: 398-407, 2004. 
14. Chu H.H., Chang Y.F. and Wang C.T., Mutations in the $\alpha$-helix directly C-terminal to the major homology region of human immunodeficiency virus type 1 capsid protein disrupt Gag multimerization and markedly impair virus particle production. J. Biomed. Sci. (this issue doi 10.1007 s11373-006-9094-6).

15. Blackburn E.H., Telomerases. Annu. Rev. Biochem. 61: 113-129, 1992.

16. Corey D.R., Telomerase inhibition, oligonucleotides, and clinical trials. Oncogene 21: 631-637, 2002.

17. Chen Y.J., Sheng W.Y., Huang P.R. and Wang T.C.V., Potent Inhibition of Human Telomerase by U-73122. J. Biomed. Sci. (this issue doi 10.1007/s11373-006-9100-z).

18. Anitha M., Gondha C., Sutliff R., Parsadanian A., Mwangi S., Sitaraman S.V. and Srinivasan S., GDNF rescues hyperglycemia-induced diabetic enteric neuropathy through activation of the PI3K/Akt pathway. J. Clin. Invest. 116: 299-302, 2006.

19. Tsai L.F., Function of GABAergic and glutamatergic neurons in the stomach. J. Biomed. Sci. 12: 255-266, 2005.

20. Britland S.T., Young R.J., Sharma A.K., Lee D., Ah-See A.K. and Clarke B.F., Vagus nerve morphology in diabetic gastropathy. Diabet. Med. 7: 780-787, 1990.

21. Li P.C., Li S.C., Lin Y.J., Liang J.T., Chien C.T. and Shaw C.F., Thoracic vagal efferent nerve stimulation evokes substance P-induced early airway bronchonstriction and late proinflammatory and oxidative injury in the rat respiratory tract. J. Biomed. Sci. 12: 671-681, 2005.

22. Lee P.G., Thomas C.H., Cai F., Regalia J. and Helke C.J., Streptozotocin-induced diabetes causes metabolic changes and alterations in neurotrophins content and retrograde transport in the cervical vagal nerve. Exp. Neurol. 170: 149-161, 2001.

23. Odekunle A., Impairment of transneuronal traffic in Streptozotocin-induced diabetes, a WGA-HRP neurohistochemical study in the rat. J. Biomed. Sci. (this issue doi 10.1007/s11373-006-9097-3).

24. Eliasson C., Sahlgren C., Berthold C.H., Stakeberg J., Celis J.E., Betsholtz C., Erilsson J.E. and Pekny M., Intermediate filament protein partnership in astrocytes. J. Biol. Chem. 274: 23996-24006, 1999.

25. Tseng W.C., Lu K.S., Lee W.C. and Chien C.L., Redistribution $\sim$ of GFAP and $\alpha$ B-crystallin after thermal stress in C6 glioma cell line. J. Biomed. Sci. (this issue doi 10.1007/ s11373-006-9091-9).
26. Swerdlow N.R., Vaccarino F.J., Amalric M. and Koob G.F., The neural substrates for the motor-activating properties of psychostimulants: a review of recent findings. Pharmacol. Biochem. Behav. 25: 233-248, 1986.

27. Mao J., Price D.D., Caruso F.S. and Mayer D.J., Oral administration of dextromethorphan prevents the development of morphine tolerance and dependence in rats. Pain 67: 361-368, 1996

28. Lange G., Action of driving stimuli from intrinsic and intrinsic sources on in situ cardiac pacemaker tissues. Circ. Res. 17: 449-459, 1965.

29. Vassalle M., Electrogenic suppression of automaticity in sheep and dog Purkinje fibers. Circ. Res. 27: 361-377, 1970.

30. Opthof T., de Jonge B., MacKaay A.J.C., Bleeker W.K., Masson-Pévet M., Jongsma H.J. and Bouman L.N., Functional and morphological organization of the guineapig sinoatrial node compared with the rabbit sinoatrial node. J. Mol. Cell. Cardiol. 17: 549-564, 1985.

31. Graziani A.T. and Vassalle M., Mechanisms underlying overdrive suppression and overdrive excitation in guinea pig sino-atrial node. J. Biomed. Sci. (this issue doi 10.1007/ s11373-006-9089-3).

32. Otterbein L.E. and Choi A.M.K., Heme oxygenase: colors of defense against cellular stress. Am. J. Physiol. 279: L1029-1037, 2000.

33. Sato K., Balla J., Otterbein L., Smith R.N., Brouard S., Lin Y., Csizmadia E., Sevigny J., Robson S.C., Vercellotti G., Choi A.M., Bach F.H. and Soares M.P., Carbon monoxide generated by heme oxygenase- 1 suppresses the rejection of mouse-to-rat cardiac transplants. J. Immunol. 166: 41854194, 2001

34. Chen Y.H., Tsai H.L., Chiang M.T. and Chau L.Y., Cardon monoxide-induced early thrombolysis contributes to heme oxygenase-1-mediated inhibition of neointimal growth after vascular injury in hypercholesterolemic mice. J. Biomed. Sci. (this issue doi 10.1007/s11373-006-9093-7).

35. Maxwell S.R.J. and Lip G.Y.H., Reperfusion injury: a review of the pathophysiology, clinical manifestations and therapeutic options. Int. J. Cardiol. 58: 95-117, 1997.

36. Park J.L. and Lucchesi B.R., Mechanisms of myocardial reperfusion injury. Ann. Thorac. Surg. 68: 1905-1912, 1998.

37. Chen S-.Y., Hsiao G., Hwang H-.R., Cheng P-.Y. and Lee Y-.M., Tetramethylpyrazine induces heme oxygenase-1 expression and attenuates myocardial ischemia/reperfusion injury in rats. J. Biomed. Sci. (this issue doi 10.1007/s11373006-9098-2) 